\title{
RESEARCH
}

\section{An Expression-independent Catalog of Genes from Human Chromosome 22}

\author{
James A. Trofatter, ${ }^{1,3}$ Kimberly R. Long, ${ }^{1,3}$ Jill R. Murrell, ${ }^{1}$ \\ Christy J. Stotler, ${ }^{1}$ James F. Gusella, ${ }^{1,2}$ and Alan J. Buckler ${ }^{1,4}$
}

Molecular Neurogenetics Unit, Departments of ${ }^{1}$ Neurology and ${ }^{2}$ Genetics, Massachusetts General Hospital and Harvard Medical School, Charlestown, Massachusetts 02129

To accomplish large-scale identification of genes from a single human chromosome, exon amplification was applied to large pools of clones from a flow-sorted human chromosome 22 cosmid library. Sequence analysis of more than one-third of the 6400 cloned products identified $35 \%$ of the known genes previously localized to this chromosome, as well as several unmapped genes and randomly sequenced cDNAs. Among the more interesting sequence similarities are those that represent novel human genes that are related to others with known or putative functions, such as one exon from a gene that may represent the human homolog of Drosophila Polycomb. It is anticipated that sequences from at least half of the genes residing on chromosome 22 are contained within this exon library. This approach is expected to facilitate fine-structure physical and transcription mapping of human chromosomes, and accelerate the process of disease gene identification.

A primary goal of the human genome initiative is the construction of fine-structure physical maps of the chromosomes in anticipation of full DNA sequence analysis. However, probably the most important purpose of this mapping, the identification and placement of human genes, can be carried out effectively before determining the complete sequence of the human genome, and can aid in increasing the resolution of the physical map. Low-resolution physical maps of human chromosomes have been described recently (Cohen et al. 1993), but considerably greater detail is needed to maximize their utility and proceed with large-scale sequencing. Identification of a representative set of genes or gene fragments corresponding to a specific genomic region would satisfy many of the requirements for finer mapping and would add a level of functional significance to these evolving maps. The resulting gene, or transcription maps, would provide a new framework for the study of structural, functional, and organizational aspects of chromosomes, and would lead to more efficient identification of genes involved in human disease. Consequently, recently the development of methods for rapid gene identification has received greater attention, and numerous strategies, including ap-

\footnotetext{
${ }^{3}$ These authors contributed equally to this work.

${ }^{4}$ Corresponding author.

E-MAlL buckler@helix.mgh.harvard.edu; FAX (617)726-5736.
}

proaches based on hybridization and biological selection (Auch and Reth 1990; Duyk et al. 1990; Buckler et al. 1991; Lovett et al. 1991; Parimoo et al. 1991), have been proposed. Exon amplification, an example of the latter category, relies on selection for functional splice sites flanking exons and thereby, avoids problematic issues such as tissue specificity or relative mRNA abundance that are inherent to other gene identification approaches. Recently, we have modified the exon amplification technique to make it applicable to the isolation of gene sequences from very complex sources of genomic DNA (Church et al. 1994). As an initial test, we have applied this method to the isolation of large numbers of gene sequences from a single human chromosome.

\section{RESULTS}

Construction of a Chromosome-specific Exon Library

Human chromosome 22 was chosen as a model for construction of exon libraries because of intensive mapping and disease gene identification efforts in this region of the genome. Plasmid DNA was prepared from pooled clones of each of the 130-microtiter plates in an arrayed cosmid library constructed from flow-sorted human chromosome 22 (LL22NC03), which represents approximately five equivalents of chromosome 


\section{CHROMOSOME 22 EXON LIBRARY}

22. An additional four pools were generated from a human-specific subset of cosmids derived from a human-hamster hybrid cell line, GM10888, containing chromosome 22 as its sole human component (Lichter et al. 1990). A total of 134 plate-pool cosmid DNAs were prepared, and each sample was digested and shotgun-cloned into the in vivo splicing vector, pSPL3 (Church et al. 1994). Plasmid DNA from pSPL3 subclones (pools of 500-2000 insert-containing clones) was transfected transiently into COS7 cells, which facilitated SV40 large T-mediated plasmid amplification and transcription. Cytoplasmic RNA derived from these transfectants was used in RNA-PCR amplifications, and the resulting products were cloned directionally as described in Methods. Exon clones ( 48 from each pool, or $\sim 6400$ clones) were arrayed, grown, and stored in 96-well microtiter plates. Approximately 24 clones from each of the first 100 pools were sequenced (a total of 2304 sequences generated) in a single pass, yielding 709 unique sequences, or an average of 7.1 unique sequences per pool. To determine the number of unique sequences in the rest of the exon library, the remaining 24 exon clones from 10 pools were sequenced and compared to all of the 709 initial sequences. An additional 40 unique sequences were produced, yielding an average of four remaining unique clones per pool. Therefore, we estimate that an average of 11.1 unique clones exist in each pool, and that sequence has been produced for $\sim 64 \%(7.1 \div 11.1)$ of the unique clones in the first 100 pools. Because there are 134 exon pools, our upper estimate of the total number of unique sequences in the entire exon library is $1487(134 \times 11.1)$. Thus, the sequences that we have generated to date represent approximately half (47\%) of those present in the library.

Complete sequence was produced for $91 \%$ of the clones analyzed, yielding a minimum average length of $\sim 125 \mathrm{bp}$ per clone. This is close to the value of $135 \mathrm{bp}$ that we have reported previously for completely sequenced sets of exons (Church et al. 1994), and likely will be similar when all the sequences are complete. We have estimated the accuracy of the sequences produced to be $-99.5 \%$, based on alignments to previously known, well-characterized gene sequences (Table 1).

An average threefold redundancy of clones corresponding to each unique sequence was observed, and is likely to be higher than this value when sequencing is complete; this may be attrib- utable to biases introduced during certain steps of the procedure. Although cultured in separate wells, growth differences among individual cosmid clones within each pool may have introduced bias during the shotgun subcloning step. However, we have designed our approach to minimize the probability that more than one exon will be isolated from the same cosmid (to maximize the uniformity of exon distribution across the chromosome). By maintaining a high complexity of target DNA (i.e., large numbers of cosmids), the growth bias is likely to be distributed across several cosmids in each pool, thus increasing the likelihood that an exon will be derived preferentially from several of the more prevalent genomic clones. Bias in the library may also be attributable to differential splicing efficiency of particular exons, as well as preferential RNA-PCR amplification or exon cloning. These aspects may be more difficult to control and are likely to be dependent on the sequence composition of exons or splice site sequences. As a result, specific exons may have a reduced probability of being trapped, but it is likely that other exons from the same gene will be identified.

\section{Sequence Data Base Comparisons}

The sequences were compared to those in public data bases (Altschul et al. 1990; BLAST comparison with Genbank and EMBL versions and updates that were available 7/23/95), and a summary of these results is presented in Tables 2 and 3. One hundred ninety-nine of the 709 sequences (28\%) analyzed are highly similar to known genes from a number of species. Included in these are 101 sequences that are identical ( $\$ 97 \%$ nucleotide identity) to segments of previously identified human genes. These can be subdivided into 48 sequences from 24 different genes that were mapped previously to chromosome 22 (in some cases, multiple exons were isolated from the same gene), and 53 other sequences corresponding to heretofore unmapped genes and expressed sequence tags (EST). Included in the latter group were sequences from RanGTPaseactivating protein 1 (Bischoff et al. 1995), phosphatidlyinositol 4-kinase (Wong and Cantley 1994), small nuclear ribonucleoprotein Sm D3 (Lehmeier et al. 1994), glutathionine $S$-transferase T1 (Pemble et al. 1994), and cadherin-13 (Tanihara et al. 1994), which are now localized provisionally to chromosome 22 . In a few cases, the sequence identity was found with the com- 
TROFATTER ET AL.

\section{Table 1. Summary of sequence data base search results}

\begin{tabular}{|c|c|c|c|c|c|c|c|c|c|c|}
\hline $\begin{array}{l}\text { Sequence } \\
\text { nurmber }\end{array}$ & Length & $\begin{array}{l}\text { Representative } \\
\text { clone }\end{array}$ & BLASTN & Species & Accession & \% SIm & BLASTX & Species & Accession & \% Sim \\
\hline \multicolumn{11}{|c|}{ Identity, mear identity, or homologue } \\
\hline $\begin{array}{l}10 \\
12\end{array}$ & $\begin{array}{r}60 \\
164\end{array}$ & ${ }_{10 H 6}^{10+46}$ & $\begin{array}{l}\text { RanGTPase activating protein } 1 \\
\text { EST07064 }\end{array}$ & HU & $\begin{array}{l}\mathbf{X 8 2 2 6 0} \\
\mathrm{T} 09171\end{array}$ & 100 & RanGAP1 & HU & $\mathrm{X82260}$ & 100 \\
\hline 13 & 206 & $10 \mathrm{~Hz}$ & merlin (NF2) & HU & L11353 & 100 & merlin (NF2) & HU & $\mathbf{S 3 3 8 0 9}$ & 100 \\
\hline 14 & 84 & 1008 & yg84a03.r1 Homo sapiens CDNA & HU & RS3636 & 96 & & 10 & ( & \\
\hline $\begin{array}{l}21 \\
25\end{array}$ & $\begin{array}{l}110 \\
138\end{array}$ & $\begin{array}{l}11 \mathrm{C10} \\
1 \mathrm{ID7}\end{array}$ & $\begin{array}{l}\text { Ig lambda light chain* } \\
\text { EST03946 }\end{array}$ & $\begin{array}{l}\mathrm{HU} \\
\mathrm{HU}\end{array}$ & $\begin{array}{l}\text { X51754 } \\
\text { T06057 }\end{array}$ & $\begin{array}{l}94 \\
90\end{array}$ & & & & \\
\hline 28 & 88 & $12 F_{2}$ & casein Inase 1-alpha & BO & M76543 & $\begin{array}{l}90 \\
69\end{array}$ & casein Innase I delta & $\mathbf{R A}$ & A46002 & 100 \\
\hline 34 & 66 & & TUPI-like enhancer of split (TUPLEI) & $\mathrm{HU}$ & $\begin{array}{l}\text { X75296 } \\
\text { D25058 }\end{array}$ & 98 & TUPI-like enhancer of sphit (TUPLE1) & HU & $\times 75296$ & 100 \\
\hline $\begin{array}{l}36 \\
37\end{array}$ & $\begin{array}{l}71 \\
69\end{array}$ & $\begin{array}{l}12 \mathrm{GS} \\
12 \mathrm{G} 10\end{array}$ & $\begin{array}{l}\text { yg } 53205.11 \text { Homo sapiens cDNA clone } \\
\text { calechol methyltransferase, upstream** }\end{array}$ & $\begin{array}{l}\text { HU } \\
\text { RA }\end{array}$ & $\begin{array}{l}\text { R25258 } \\
\text { Z12652 }\end{array}$ & $\begin{array}{r}100 \\
85\end{array}$ & glutathione reductase & Pf & $\mathbf{X 8 3 6 0 3}$ & 78 \\
\hline 40 & 284 & $39 \mathrm{E} 7$ & yd18f06.s1 Homo sapiens CDNA clone & HU & T69835 & 92 & & & & \\
\hline 42 & 144 & & fibu in A,B,C,D (FBLN1) & HU & $\begin{array}{r}X 53743 \\
\mathbf{X} 97713\end{array}$ & 100 & fibalin A,B,C,D (FBLN1) & HU & X53743 & 100 \\
\hline $\begin{array}{l}48 \\
59\end{array}$ & $\begin{array}{c}102 \\
67\end{array}$ & $\begin{array}{l}13 \mathrm{HA} \\
\text { 14A9 }\end{array}$ & $\begin{array}{l}\text { fibulin A,B,C,D (FBLN1) } \\
\text { ye81 c04,s1 Homo sapiens cDNA }\end{array}$ & $\underset{H U}{H U}$ & $\begin{array}{l}\text { XS3743 } \\
\text { R01350 }\end{array}$ & $\begin{array}{l}100 \\
100\end{array}$ & fibulin $A, B, C, D(F B L N 1)$ & HU & X53743 & 100 \\
\hline 79 & 36 & 15FI1 & yt30c02.t1 Homo sapiens cDNA & HU & R24354 & 100 & & & & \\
\hline 80 & 153 & 16A1 & DGCR2 & HU & $\mathrm{X84076}$ & 100 & DGCR2 & HU & X84076 & 100 \\
\hline $\begin{array}{r}86 \\
100\end{array}$ & $\begin{array}{r}111 \\
39\end{array}$ & $\begin{array}{l}\text { 16B2 } \\
\text { 19E1 }\end{array}$ & $\begin{array}{l}\text { phosphatidylinositol transfer protein } \\
\text { peroxisome proliferator activated recept. }\end{array}$ & $\underset{H U}{H U}$ & $\begin{array}{l}\text { M73704 } \\
\text { L02932 }\end{array}$ & $\begin{array}{r}77 \\
100\end{array}$ & phosphatidylinositol transfer prodeln & HU & Q00169 & 86 \\
\hline 101 & 130 & 19E2 & Ewing sarcoma (EWSR1) & HU & X66899 & 100 & Ewing garcoma (EWSR1) & HU & X66899 & 100 \\
\hline 107 & 58 & 19F7 & cotechol - O-methyltransforase (COMT) & HU & M65212 & 100 & calectoo-0-methyltransferase & HU & M65212 & 100 \\
\hline 117 & 117 & $\begin{array}{l}20 \mathrm{~A}^{2} \\
21 \mathrm{E} 3\end{array}$ & $\begin{array}{l}\text { yg590009.r1 Homo sapiens cDNA } \\
\text { mettin (NF2) }\end{array}$ & HU & $\begin{array}{l}\mathrm{R} 35102 \\
\mathrm{~L} 1353\end{array}$ & $\begin{array}{l}100 \\
100\end{array}$ & & & & \\
\hline $\begin{array}{l}128 \\
137\end{array}$ & $\begin{array}{l}123 \\
136\end{array}$ & $\begin{array}{l}21 E 3 \\
21 F 3\end{array}$ & $\begin{array}{l}\text { mettin (NF2) } \\
\text { GM-CSP reoctor beta chain (CSF2RB) }\end{array}$ & HU & $\begin{array}{l}L 11353 \\
159941\end{array}$ & $\begin{array}{l}100 \\
98\end{array}$ & $\begin{array}{l}\text { mertin (NF2) } \\
\text { GM-CSF receptor beta chain (CSF2RB) }\end{array}$ & HU & $\mathbf{\$ 3 3 8 0 9}$ & 100 \\
\hline $\begin{array}{l}137 \\
141\end{array}$ & $\begin{array}{r}136 \\
56\end{array}$ & $21 \mathrm{F10}$ & $\begin{array}{l}\text { GM-CSP receptor beta chain (CSF2RB) } \\
\text { CDNA clone seq1 } 298\end{array}$ & HU & $\begin{array}{l}\text { M59941 } \\
\text { T10083 }\end{array}$ & 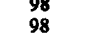 & & HU & M59941 & 99 \\
\hline 145 & 249 & $22 \mathrm{A4}$ & ym2007.s1 Homo sapiens cDNA & HU & H15984 & 95 & aconitase & $\mathbf{P I}$ & P16276 & 96 \\
\hline 158 & 75 & $24 A 7$ & yt83a06.s1 Homo sapiens cDNA & HU & R33473 & 100 & ( & $M$ & Moro & 30 \\
\hline 169 & 136 & $26 \mathrm{A5}$ & fibuin A,B,C,D (FBLNI) & HU & X53743 & 100 & fibulin A,B,C,D (FBLN1) & HU & X53743 & 100 \\
\hline 171 & 137 & $20 \mathrm{B1}$ & ya53d06.s1 Homo sapiess cDNA & HU & T67209 & 100 & probable glutathione reductase & Ce & P30635 & 66 \\
\hline $\begin{array}{l}172 \\
174\end{array}$ & 206 & 2082 & partial cDNA sequenco; clone a-21605 & HU & $\begin{array}{l}\text { Z45264 } \\
\text { R11814 }\end{array}$ & 99 & & & & \\
\hline $\begin{array}{l}174 \\
175\end{array}$ & $\begin{array}{r}154 \\
75\end{array}$ & $\begin{array}{l}28 \mathrm{Bg} 9 \\
28 \mathrm{Al}\end{array}$ & $\begin{array}{l}\text { ye53.05s.s1 Homo saplems cDNA } \\
\text { EST02272 }\end{array}$ & $\begin{array}{ll}\mathrm{HU} \\
\mathrm{HU}\end{array}$ & $\begin{array}{l}\text { R11814 } \\
\text { M85753 }\end{array}$ & $\begin{array}{r}96 \\
100\end{array}$ & & & & \\
\hline 177 & 204 & $28 \mathrm{~A} 6$ & $\begin{array}{l}\text { EST02272 } \\
\text { EST02272 }\end{array}$ & HU & M85753 & 98 & & & & \\
\hline 180 & 141 & $29 \mathrm{E} 3$ & Ig lambda chain variable & HU & M94113 & 100 & & & & \\
\hline 182 & 127 & 29E8 & ye98f07 s1 Homo sapiens cDNA & HU & R07694 & 100 & & & & \\
\hline 188 & 112 & $2 \mathrm{EA}$ & Human bomologue of yeast sec7 & HU & M85169 & 77 & Human homologue & HU & $\mathbf{S 2 4 1 6 8}$ & 97 \\
\hline 192 & 116 & $2 \mathrm{~F} 6$ & chloride chamel & HU & $\mathrm{x} 77197$ & 89 & chloride charnel & $\mathbf{H U}$ & $x 77197$ & 97 \\
\hline $\begin{array}{l}211 \\
212\end{array}$ & 193 & $32 \mathrm{AI}$ & & HU & FO7861 & $\begin{array}{r}96 \\
100\end{array}$ & & & & \\
\hline $\begin{array}{l}212 \\
220\end{array}$ & $\begin{array}{l}193 \\
123\end{array}$ & $\begin{array}{l}32 \mathrm{~A} 2 \\
32 \mathrm{BB}\end{array}$ & Etal-kung CDNA S'-end sequence & HU & D31239 & 100 & period clock protein & MU & P08399 & 85 \\
\hline 228 & 94 & $33 \mathrm{~F} 4$ & yi02e10.s1 Homo sapiens cDNA & HU & R53774 & 96 & & & & \\
\hline 235 & 111 & 36A1 & ganmm-ghtanyylteransferase rehted & HU & M64099 & 100 & gamma-glutamyltransferase related & HU & A41125 & 100 \\
\hline $\begin{array}{l}251 \\
258\end{array}$ & 100 & $39 \mathrm{E} 2$ & breakpoint cluster region (BCR) & HU & U07000 & 100 & & & & \\
\hline $\begin{array}{l}258 \\
265\end{array}$ & $\begin{array}{l}165 \\
118\end{array}$ & 3B12 & $\begin{array}{l}\text { yfl13805sl Homo sapiens cDNA } \\
\text { partial cDNA sequence; done c-10d08 }\end{array}$ & HU & $\begin{array}{l}\text { R070106 } \\
\text { Z39110 }\end{array}$ & $\begin{array}{r}100 \\
92\end{array}$ & alpha-actinid & CH & $\mathbf{S 4 5 6 7 3}$ & 62 \\
\hline 273 & 184 & $40 \mathrm{~A} 10^{\circ}$ & 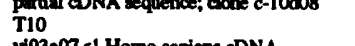 & $\mathbf{M U}$ & $\times 74504$ & 86 & Tio & MU & X74504 & 100 \\
\hline $\begin{array}{l}282 \\
283\end{array}$ & $\begin{array}{l}63 \\
96\end{array}$ & $\begin{array}{l}41 \mathrm{~EB} \\
41 \mathrm{Eg}\end{array}$ & $\begin{array}{l}\text { yi93207.11 Homo sapiens cDNA } \\
\text { TUP1-like enhancer of solit (TUPLE })\end{array}$ & $\underset{H U}{H U}$ & $\begin{array}{l}\mathrm{RBO434} \\
\times 75206\end{array}$ & $\begin{array}{r}87 \\
100\end{array}$ & TUP1-like enhanoer of split (TUPLEI) & & & \\
\hline $\begin{array}{l}283 \\
284\end{array}$ & 96 & $41 \mathrm{E}_{11}$ & $\begin{array}{l}\text { TUP1-Hike enhance of (split (TUPLEI) } \\
\text { ye83.05.s1 Homo sappeas cDNA }\end{array}$ & HU & 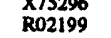 & $\begin{array}{l}100 \\
100\end{array}$ & R01H2.6 gene product & Ce & $\begin{array}{l}\mathbf{X} 75296 \\
\mathbf{U} 00035\end{array}$ & $\begin{array}{r}100 \\
71\end{array}$ \\
\hline 285 & 230 & 41FI & Ewing sarcooma (EWSRI)* & HU & $\mathbf{X 6 6 8 9 9}$ & 99 & & & & \\
\hline 287 & 60 & $41 \mathrm{F3}$ & TUP1-Hike enhancer of aptit (TUPLE1) & HU & $X 75296$ & 100 & & & & \\
\hline 291 & 70 & 42A7 & cellular my ooin beary chain (MYH9) & HU & M81105 & 100 & cellular myosin heavy chain & HU & A34876 & 100 \\
\hline 300 & 113 & 43E4 & GM-CSF receptor beta chain (CSF2RB) & HU & M59941 & 100 & GM-CSF receptor bela chain (CSF2RB) & HO & P32927 & 100 \\
\hline $\begin{array}{l}301 \\
305\end{array}$ & 144 & $9 / 44$ & His 594-f Homo sapiens cDNA & HU & R41006 & $\begin{array}{l}96 \\
90\end{array}$ & & & & \\
\hline $\begin{array}{l}305 \\
307\end{array}$ & $\begin{array}{l}105 \\
220\end{array}$ & 43F3 & D4S2A63 homeobox-like gene & HU & U18977 & $\begin{array}{r}90 \\
100\end{array}$ & phosphatidylinositol 4-kinase & HU & L36151 & \\
\hline $\begin{array}{l}307 \\
310\end{array}$ & $\begin{array}{l}220 \\
191\end{array}$ & $\begin{array}{l}43 \mathrm{~F} 6 \\
43 \mathrm{~F} 12\end{array}$ & phosphatidy linositol 4-kinase & HU & L36151 & $\begin{array}{r}100 \\
96\end{array}$ & asparagine sypthetase & Hu & & 100 \\
\hline $\begin{array}{l}310 \\
312\end{array}$ & 128 & $\begin{array}{l}43 \mathrm{~F} 12 \\
44 \mathrm{~A}^{3}\end{array}$ & $\begin{array}{l}\text { separaging esynthetase } \\
\text { UK-HGMP seguence ID A A A ASTE }\end{array}$ & $\begin{array}{l}\text { HU } \\
\text { HU }\end{array}$ & $\begin{array}{l}\text { M27396 } \\
\text { Z19815 }\end{array}$ & $\begin{array}{l}96 \\
88\end{array}$ & & & A2/443 & 97 \\
\hline 326 & 149 & $45 \mathrm{E} 6$ & $\begin{array}{l}\text { UK-HGMP sequence DD AAAASTE } \\
\text { ESTOS647 }\end{array}$ & HU & $\begin{array}{l}\text { Z19815 } \\
\text { T07757 }\end{array}$ & $\begin{array}{l}88 \\
99\end{array}$ & casein Wuase I delta & RA & 006486 & 100 \\
\hline 329 & 251 & 45F3 & beta-crystallin snbunit beta BI & Do & $X 01808$ & 86 & beta-crystallin subunit beta BI & BO & 507264 & 93 \\
\hline 332 & 204 & 45F12 & fbulin D (FBLNI) & HU & 001244 & 99 & fibulin D (FBLNI) & HU & U01244 & 100 \\
\hline 333 & 126 & 46Al & STS UT6047 & HU & L30702 & 99 & & & & \\
\hline 338 & 150 & 47AB & yg02g12s1 Homo sapiens CDNA & HU & $\mathbf{R} 17513$ & 98 & & & & \\
\hline $\begin{array}{l}343 \\
352\end{array}$ & $\begin{array}{l}136 \\
76\end{array}$ & $\begin{array}{l}4789 \\
48 \mathrm{~F} 7\end{array}$ & & $\underset{\mathbf{H U}}{\mathbf{H U}}$ & $\begin{array}{l}778985 \\
\text { T08420 }\end{array}$ & $\begin{array}{l}100 \\
100\end{array}$ & & & & \\
\hline 360 & 218 & $4 F_{3}$ & $\begin{array}{l}\text { ESTO63i1 } \\
\text { thyroxine delodilinase }\end{array}$ & $\mathbf{R A}$ & $\begin{array}{l}T 08420 \\
\text { M21476 }\end{array}$ & 89 & thyroxine delodinase & RA & P13700 & 95 \\
\hline $\begin{array}{l}364 \\
368\end{array}$ & $\begin{array}{r}53 \\
125\end{array}$ & 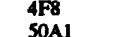 & you43e06,rl Homo sapiens cDNA & HU & H18565 & 87 & & & & \\
\hline 375 & 114 & $\begin{array}{l}\text { SOAI } \\
\text { SOB12 }\end{array}$ & ye64b05.sI Homo sapiens cDNA & HU & T99352 & 100 & $\begin{array}{l}\text { T10 } \\
\text { collapen alpha } 3(\mathrm{VI}) \text { chain }\end{array}$ & $\underset{\mathrm{HU}}{\mathrm{MU}}$ & $\begin{array}{l}\mathrm{X} 74504 \\
\mathbf{S} 3679\end{array}$ & $\begin{array}{l}100 \\
100\end{array}$ \\
\hline 383 & & 508 & $L-2 R$-beta & HU & A07797 & 100 & L-2R-beta & HU & A07795 & 100 \\
\hline 390 & 184 & GB6 & Yg73708,s1 Homo sapiens cDNA & HU & R51689 & 97 & & MU & P28658 & 94 \\
\hline 395 & $\begin{array}{r}82 \\
140\end{array}$ & $\begin{array}{l}7 G 7 \\
7 G 10\end{array}-10$ & beta-alanine systhase & RA & M97662 & $\begin{array}{l}88 \\
95\end{array}$ & beta-alandne synthase & RA & Q03248 & 100 \\
\hline $\begin{array}{l}396 \\
400\end{array}$ & $\begin{array}{l}140 \\
176\end{array}$ & $\begin{array}{l}7 \mathrm{~F} 10 \\
\mathrm{BD} 12\end{array}$ & $\begin{array}{l}\text { break point cluster gene (BCR) } \\
\text { CDNA clone B270 }\end{array}$ & $\underset{\text { HU }}{\mathrm{HU}}$ & $\begin{array}{l}\text { Yoo } \\
\text { T200 }\end{array}$ & 100 & break point cluster gene (BCR) & HU & U07000 & 95 \\
\hline $\begin{array}{l}400 \\
409\end{array}$ & $\begin{array}{l}176 \\
174\end{array}$ & 9A10 & $\begin{array}{l}\text { DDNA clone B270 } \\
\text { SnRNP core protein Sm D3 }\end{array}$ & Hu & $\begin{array}{l}T 20010 \\
\text { U15009 }\end{array}$ & $\begin{array}{l}100 \\
100\end{array}$ & & & & \\
\hline $\begin{array}{l}409 \\
415\end{array}$ & $\begin{array}{l}174 \\
145\end{array}$ & 9H12 & $\begin{array}{l}\text { SuRNP core protein Sm D3 } \\
\text { o-sis (PDGFB) }\end{array}$ & HU & $\begin{array}{l}015099 \\
K 01916\end{array}$ & 100 & $\begin{array}{l}\text { SoRNP core protein Sm D3 } \\
\text { platele-derived growth factor B }\end{array}$ & HU & U15009 & 100 \\
\hline $\begin{array}{l}415 \\
417\end{array}$ & $\begin{array}{l}145 \\
168\end{array}$ & 100E1 & $\begin{array}{l}\text { C-sis (PDGFB) } \\
\text { yo77d12.r1 Homo sapiens cDNA }\end{array}$ & HU & $\begin{array}{l}\text { Koly } \\
\text { H30621 }\end{array}$ & 100 & platelet-derived growth factor B & HU & $\mathbf{X} 00561$ & 100 \\
\hline $\begin{array}{l}417 \\
419\end{array}$ & $\begin{array}{r}168 \\
95\end{array}$ & 100E3 & $\begin{array}{l}\text { yo77d12.r1 Homo sapiens cDNA } \\
\text { MDB1128R Mus musculus cDNA }\end{array}$ & MU & R75186 & 92 & & & & \\
\hline 421 & III & $100 \mathrm{E}$ & & HU & $\mathrm{Z} 23024$ & 73 & thoGAP prolein & HU & S34296 & 91 \\
\hline 430 & 206 & $100 \mathrm{~F} 8$ & EST00943 & HU & M78795 & 100 & & & & \\
\hline 440 & 50 & 27 F8 & NMDA receptor NR2C subunit* & $\mathbf{R A}$ & M91563 & 91 & & & & \\
\hline 466 & 190 & $52 \mathrm{~A} 12$ & UK-HGMP sequence ID AAADFQK & FU & $\mathrm{Z} 20962$ & 99 & & & & \\
\hline 470 & 122 & 52811 & inmunoglobulin lambda-Hike (IGLL) & $\mathbf{H U}$ & $\mathrm{X} 52204$ & 100 & & & & \\
\hline 471 & 96 & 52812 & ESTO45 & $\mathbf{H O}$ & T06655 & 89 & & & & \\
\hline 473 & 141 & 53E3 & ras inhibitor & HU & M37191 & 90 & Ras inhibitor & HU & C38637 & 100 \\
\hline $\begin{array}{l}474 \\
491\end{array}$ & 101 & 53E4 & ye81 c04 ,1 Homo sapiens cDNA & FU & R01350 & 100 & & & & \\
\hline 495 & $\begin{array}{l}154 \\
138\end{array}$ & $\begin{array}{l}54 \mathrm{AS} \\
\text { 54B1 }\end{array}$ & $\begin{array}{l}\text { partial cDNA sequence; clone c-1 valog } \\
\text { partial cDNA sequence; clone c- c-0g } 0009\end{array}$ & $\underset{H U}{\text { HW }}$ & $\begin{array}{l}\text { FO7091 } \\
\text { ZA2345 }\end{array}$ & $\begin{array}{r}100 \\
97\end{array}$ & & & & \\
\hline
\end{tabular}


Table 1. (continued)

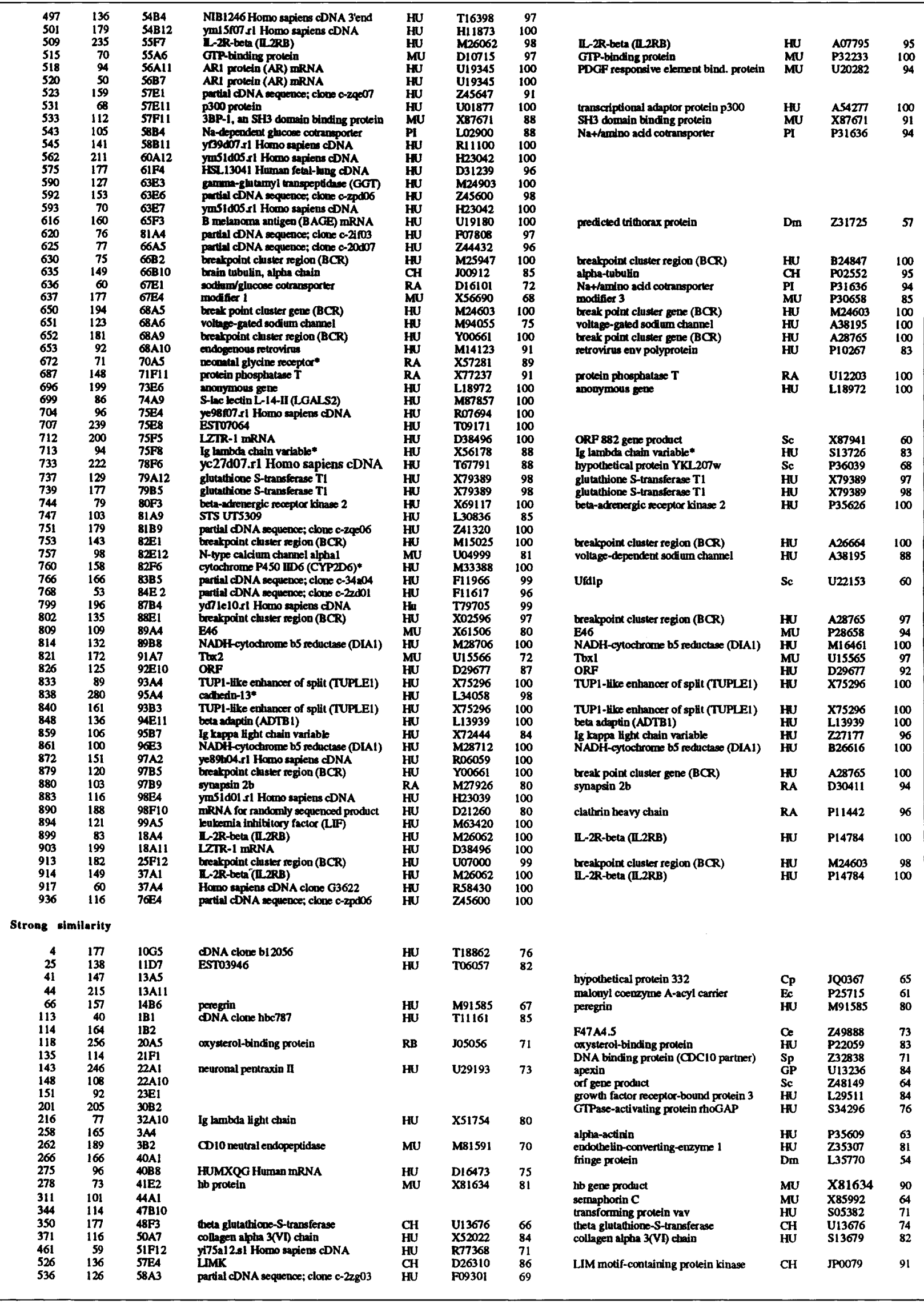


TROFATIER ET AL.

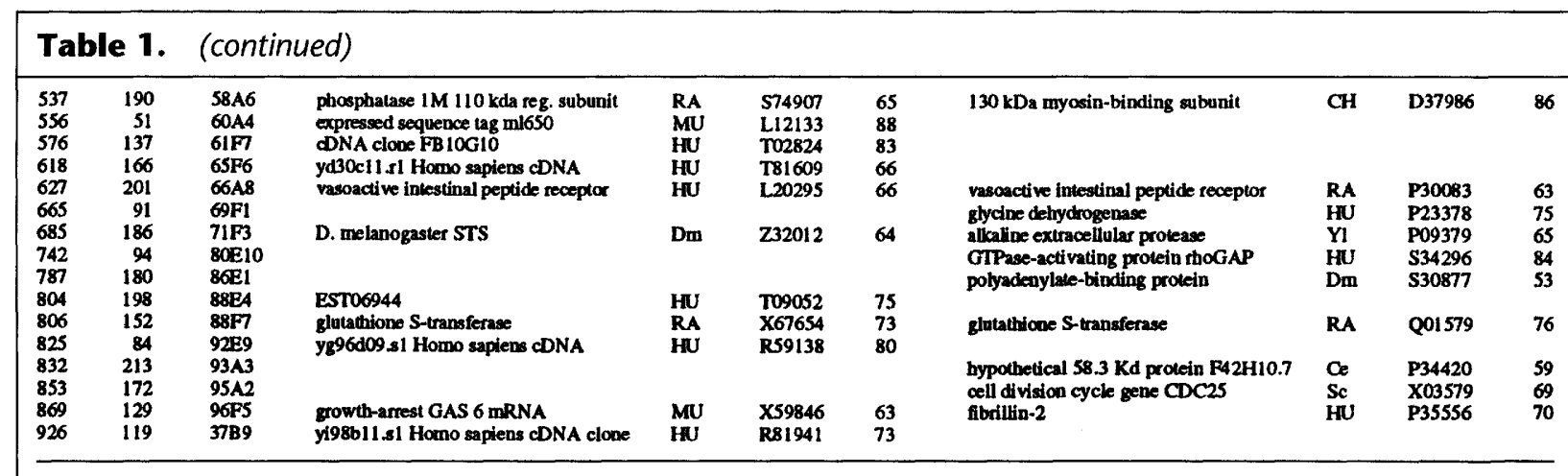

The criteria for categorizing similarities were based on BLASTN or BLASTX results (Altschul et al. 1990) and were as follows: Identity, near identity, or homolog, nucleic acid, or protein similarities $\geqslant 85 \%$ and $P$ value $\leqslant 10^{-5}$; strong similarity, nucleic acid, or protein similarity $\geqslant 50 \%$ and $P$ value $\leqslant 10^{-3}$. Asterisks denote sequence similarity to the complementary strand of the data base "hit." Species names: (BO) bovine; (Ce) Caenorhabditis elegans; (CH) chicken; (Cp) Clostridium perfringens; (Dd) Dictyostelium discoideum; (Dm) Drosophila melanogaster; (Ec) Escherichia coli; (GP) Cavia porcellus (guinea pig); (HU) human; (MU) mouse; (PI) pig; (RA) rat; (RB) rabbit; (Sc) Saccahromyces cerevesiae; (Sp) Schizosaccharomyces pombe; (YI) Yarrowia lipolytica.

plementary strand of known genes. The most notable of these were the matches of sequences 285 and 760 , which were identical to the complementary strands of Ewing sarcoma and cytochrome P450 IID6 gene sequences, respectively. In both cases, the match was found near the $3^{\prime}$ end of the mRNA sequences of these genes, and the sequences flanking the aligned regions closely match consensus splice sites. Whether these sequences represent artifacts or genes encoded on the overlapping DNA strand opposite to the known genes remains unclear.

The remaining 98 sequences represent human homologs of genes from other species, members of gene families, or genes sharing strong similarities with known genes. Among the more interesting sequences with similarities are those that represent novel human genes that are related to others with known or putative functions. For example, the predicted amino acid se- quence of exon 637 is highly similar to part of a common domain, termed the chromodomain, found in genes whose products associate with heterochromatin James and Elgin 1986; Paro and Hogness 1991; Singh et al. 1991; Delmas et al. 1993). The best studied of these genes are Drosophila heterochromatin-associated protein, HP1, and Polycomb. Both of these genes have been shown to control, by repression, developmental regulators such as homeotic genes. The chromodomain appears to be essential for assembly of these proteins into chromatin as part of a multiple protein complex, as mutations or deletions in this domain in the Polycomb protein abolish its ability to associate with heterochromatin (Messmer et al. 1992). Thus, the sequence represented by exon 637 may represent a novel regulator of homeotic function in human development. Figure 1 is an amino acid alignment of exon 637 with the chromodomains of other pro-

Table 2. Summary of sequence data base comparisons

\begin{tabular}{lcc}
\hline \multicolumn{1}{c}{ Category } & Number of sequences & Percent of total \\
\hline Identity to known human genes/ESTs & 101 & 14.2 \\
Near identity or strong similarity & 98 & 13.8 \\
Weak or no similarity & 404 & 57.0 \\
Repetitive sequence/artifact & 106 & 15.0 \\
Total unique sequences & 709 & 100.0 \\
\hline Sequences have been placed into each category based on the results of BLASTN and BLASTX comparisons \\
(Altschul et al. 1990). Criteria for similarity categories are described in the legend to Table 1. \\
\hline
\end{tabular}


Table 3. Chromosome 22 gene sequences identified

\begin{tabular}{|c|c|c|c|}
\hline $\begin{array}{l}\text { Locus } \\
\text { name }\end{array}$ & Gene name & $\begin{array}{l}\text { Chromosomal } \\
\text { position }\end{array}$ & $\begin{array}{l}\text { Number of } \\
\text { matching } \\
\text { sequences }\end{array}$ \\
\hline ADRBK2 & $\beta$-adrenergic receptor kinase & q11 & 1 \\
\hline ADTB1 & $\beta$-adaptin & $\mathrm{q} 12$ & 1 \\
\hline$B C R$ & breakpoint cluster region & $\mathrm{q} 11.2$ & 7 \\
\hline COMT & catechol-O-methyltransferase & $\mathrm{q} 11.2$ & 1 \\
\hline CSF2RB & GM-CSF receptor, $\beta$-chain & q13 & 2 \\
\hline CYP2D6 ${ }^{\mathrm{a}}$ & cytochrome P450 IID6 & $q 13$ & 1 \\
\hline DIA1 & NADH-cytochrome b5 reductase & q13.3 & 2 \\
\hline $\mathrm{EWSR}^{\mathrm{a}}$ & Ewing sarcoma & $\mathrm{q} 12.1-\mathrm{q} 12.2$ & 2 \\
\hline FBLN1 & fibulin-1 (isoforms A, B, C, D) & $\mathrm{q} 13.2-\mathrm{q} 13.3$ & 4 \\
\hline GGT & $\gamma$-glutamyl transpeptidase & $\mathrm{q} 11.2$ & 1 \\
\hline GGT-rel & GGT-related & $\mathrm{q} 11.2$ & 1 \\
\hline IGLL8 & immunoglobulin $\lambda$-like & $\mathrm{q} 11.2$ & 1 \\
\hline IGLV & immunoglobulin light-chain variable & $\mathrm{q} 11.2$ & 1 \\
\hline IL2RB & interleukin 2 receptor, $\beta$-subunit & $q 13$ & 2 \\
\hline LGALS2 & S-lac lectin L-14-II & $q 12.2-q 13$ & 1 \\
\hline LIF & leukemia inhibotoory factory & q12 & 1 \\
\hline MYH9 & cellular myosin heavy chain & $\mathrm{q} 12.3-\mathrm{q} 13.1$ & 1 \\
\hline NF2 & merlin, neurofibromatosis type 2 & $\mathrm{q} 12.1-\mathrm{q} 12.2$ & 2 \\
\hline PDGFB & c-sis, platelet-derived growth factor & q13 & 1 \\
\hline PPAR & peroxisome proliferation activated receptor & $q 12-q 13.1$ & 1 \\
\hline \multirow[t]{5}{*}{ TUPLE1 } & TUP1-like enhancer of split & q11 & 5 \\
\hline & anonymous gene & q12 & 1 \\
\hline & p300 transcription adaptor & q13.2 & 1 \\
\hline & LZTR-1 & q11 & 2 \\
\hline & AR1 & $?$ & 2 \\
\hline
\end{tabular}

teins. Exon 637, however, does not contain the complete chromodomain, but begins several amino acids downstream and continues beyond the carboxyl end of the motif. Interestingly, the genomic structure of the Polycomb locus of Drosophila has been determined, and the $5^{\prime}$ end of exon 637 is at the precise location of an intron-exon boundary within this gene (Paro and Hogness 1991). This suggests that some phylogenetic conservation of genomic structure exists for the 637 gene or that the 637 gene may represent the human homolog of Polycomb.

In addition to exon 637 , several of the sequences appear to be closely related to genes involved in growth regulatory, developmental, or cell type-specific-processes. Isolation of these types of genes, many of which are likely to be representative of low-abundance or tissue- specific mRNA species exemplifies an advantage of the exon amplification approach: expressionindependent gene identification. The overwhelming majority of human genes are expressed at low levels, producing low-abundance mRNA (Hastie and Bishop 1976). Many other approaches that require significant levels of gene expression, or knowledge of tissue specificity, may fail to identify such genes with any efficiency. This includes most large-scale random cDNA sequencing strategies (Adams et al. 1991, 1992; Khan et al. 1992; Okubo et al. 1992), which are biased toward identification of mRNAs that are highly expressed in the tissue (or cells) from which the library was generated, as well as approaches using RNA derived from monochromsomal- or region-specific human-rodent hybrid cell lines (Liu et al. 1989; Corbo et al. 1990; Jones 


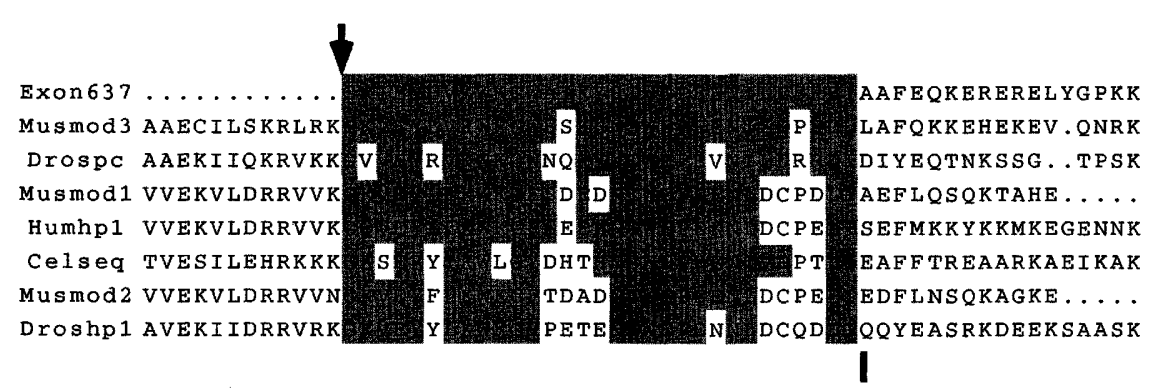

"Chromodomain"

Figure 1 Alignment of the predicted peptide sequence of 637 with other chromodomain-containing proteins. Identities or conservative amino acid differences between 637 and each protein are shaded. The arrow indicates the location of an intron in the Drosophila melanogaster Polycomb gene. (Musmod3) Mouse modifier-3 (accession no. P30658); (Drospc) Drosophila melanogaster Polycomb (accession no. P26017); (Musmod1) mouse modifier-1 (accession no. P23197); (Humhp1) human HP1 homolog (accession no. S62077); (Celseq) Caenorhabditis elegans, hypothetical 33.8-kD protein (accession no. P34618); (Musmod2) mouse modifier-2 (accession no. P23198); (Droshp1) Drosophila melanogaster heterochromatin protein HP1 (accession no. P05205).

et al. 1992). Direct or cDNA selection procedures (Parimoo et al. 1991; Lovett et al. 1991) have been designed to minimize this problem of representation by enriching for rare mRNAs and complement exon amplification in that they can enrich for these low abundance species, if they are present. These approaches are also adaptable for en masse, region-specific gene identification, as Del Mastro et al. (1995) demonstrate using human chromosome 5 as a target.

One hundred six of the 709 sequences $(-15 \%)$ were artifacts or similar to repetitive elements. The artifacts were derived from a number of sources but originate primarily from pSPL3 and the pLawrist16 cosmid vector. The higher prevalence of these clones as artifacts in these assays, as compared to assays of single cosmid clones, may be attributable to the vast molar excess of these sequences relative to the genomic sequences targeted for exon isolation. It should be noted that sequences from pSPL3 or pLawrist comprise $\sim 20 \%$ (10\% each) of all clones in the library, based on sequencing of -2300 clones. These can be eliminated readily by hybridization detection, thereby significantly reducing the effort required for sequencing of similar libraries.

\section{Localization of Exons to Chromosome 22}

The starting genomic DNA for these experiments was derived from flow-sorted chromosomes de- rived from a human-hamster hybrid that contains preferentially human chromosome 22 , but also retains chromosomes 9 and $\mathrm{Y}$ at a low frequency. Thus, the possibility exists that some of the exons originate from nonchromosome 22 genomic DNA, including hamster. This estimate has been confirmed by mapping these sequences to Southern blots of human, hamster, and GM10888 (monochromosome 22 human-hamster hybrid) DNAs (data not shown). Of 21 randomly chosen exons that hybridized to the blots, 17 (81\%) mapped to chromsome 22,3 were of hamster origin, and 1 was human, but apparently not originating from chromosome 22 . These numbers are consistent with the estimated nonchomosome 22 content of the starting cosmid library. The percentage of chromosome 22-specific sequences is likely to be higher, as we excluded from our analysis exons from previously mapped genes.

\section{DISCUSSION}

The collection of clones described above represents one of the first large-scale, chromosomespecific isolations of human gene sequences, and will serve as an inroad to the development of an integrated physical and transcription map of human chromosome 22 . It is likely to be an invaluable tool for creating the high resolution maps that are needed for sequencing of the human genome. Extrapolation of our results leads to a prediction that $\sim 1500$ sequences will be generated after identification of all unique sequences in this collection of clones (of which $>1200$ will be nonrepetitive and nonartifact), and an estimation that nearly half of these sequences have been produced to date. In this study 24 of $59(41 \%)$ of the fully sequenced genes (nonpseudogenes) currently known to map to chromosome 22 were identified, suggesting that a similar percentage of all genes on this chromosome are represented by the sequences that have been generated thus far. Therefore, it is anticipated that exons from as many as $80 \%$ of the genes on this chromosome 


\section{CHROMOSOME 22 EXON LIBRARY}

will be represented in this library after completion of library sequencing. To further increase the representation of genes in this library and eliminate much of the bias in gene identification, subsequent isolation of exons will be performed on cosmids that stochastically failed to produce exons in the initial library construction.

Several aspects of this approach make it ideal for construction of detailed physical/ transcription maps. First, the use of genomic DNA from a specific human chromosome allows positional information to be associated provisionally to each exon, circumventing the need to localize cloned gene fragments that have been isolated and sequenced as random cDNA, and provides a more direct approach to saturate specific regions of the genome with such sequences. It should be noted, however, that sources such as flow-sorted chromosomes, or libraries constructed from them, frequently originate from human-rodent somatic cell hybrids; a small fraction of genomic DNA, hence exons, may originate from the rodent parent or from other human chromosomes present within the hybrid. The chromosome 22 cosmid library used in this study was derived from a hybrid cell line that also retains human chromosomes 9 and $\mathrm{Y}$ at a low frequency. We have estimated that $10 \%-20 \%$ of the exon library contains sequences from genomic DNA not originating from human chromosome 22, the majority of which is from hamster. Thus, although the exon library is highly enriched for chromosome 22 gene sequences, it is not pure, and the sequences are annotated as such. It should be noted, however, that no exons from known genes that map to any other human chromosome were identified to date in these studies, whereas 48 exons from chromosome 22specific genes were isolated. In addition, the recent availability of high-quality monochromosomal hybrids for most human chromosomes, coupled with improved flow-sorting will reduce dramatically the problem for future exon library constructions.

Second, the exons produced by this procedure are consummate multi-purpose mapping reagents. Because the vast majority of exons are single copy sequences, they can be used as hybridization probes in filter-based mapping procedures. Moreover, we have found that exon sequences are easily converted to sequence-tagged sites (STS) for use in PCR-based mapping schemes (Green and Olson 1990). With an average spacing of $60-70 \mathrm{~kb}$, the chromosome 22 exons iden- tified thus far could be used to complete the $\mathrm{Hu}$ man Genome Initiative's goal of one STS per 100 $\mathrm{kb}$ for each chromosome. The conversion of exons to cDNAs would then provide both ordering and orientation across groups of yeast artificial chromosomes (YACs) or cosmids as well as confirm any existing contig information. Also, the cross-species conservation of many exons allows for effective comparative mapping of genes and for direct comparison of emerging physical maps in humans, mice, and other model genomes.

Third, because exon amplification is not dependent on the level or pattern of expression of the gene that is isolated, representational biases inherent in tissues or cells from which cDNA libraries are constructed, are eliminated. Exons can be used as a DNA sequence source for determining the specific expression pattern of the gene from which it originated by using quantitative assays of RNA expression, such as Northern blotting, S1 nuclease, or RNase protection, in situ hybridization, or by using PCR to detect the presence of exon sequences in a cDNA library (Church et al. 1993; Munroe et al. 1995). The resulting information allows for effective screening of appropriate cDNA libraries to saturate quickly a given genomic region with genes. This property of the technique has already been applied successfully to positional cloning efforts in Huntington's disease and neurofibromatosis 2 (Huntington's Disease Collaborative Research Group 1993; Trofatter et al. 1993), resulting in identification of the disease genes by isolation of 28 and 8 of their exons, respectively, as well as successful effort to identify several other human and mouse disease genes (Vidal et al. 1993; Vulpe et al. 1993; Walker et al. 1993; Cachon-Gonzalez et al. 1994; Hästbacka et al. 1994).

The large-scale exon isolation approach that we have applied here is currently being transferred to several other human chromosomes. Our data suggest that this method could identify segments from the majority of human genes before to the generation of the human genome's sequence. Moreover, the strategy would help to achieve this goal by facilitating the necessary construction and comparison of fine structure physical maps while simultaneously integrating them into transcription maps of greater utility to a wide range of researchers in genetics and biology. Continued application of this strategy represents a most effective and cost-efficient means of substantiating the most prominent rationale for pursuing the Human Genome Initiative, cre- 
TROFATTER ET AL.

ation of the infrastructure needed to support the rapid and efficient discovery of genes causing human disease.

\section{METHODS}

\section{Exon Library Construction}

Exon amplification was performed as described (Church et al. 1994), with some modification. Cosmid-containing clones were propagated in 96-well microtiter plates, pooled, and cosmid DNA purified using the alkaline lysis method. Before propagation, cosmids containing ribosomal gene DNA (rDNA) were identified by hybridization and removed from each plate. This was done to insure against overrepresentation of chromosome 22 rDNA sequences in the amplified and cloned products. Shotgun cloning into pSPL3, transfections, and RNA isolations were performed as described (Church et al. 1994). Initially, RNA-based-PCR amplification (RT-PCR) and cloning of the resulting products was performed as described (Church et al. 1994), but was replaced by ligation-independent cloning using uracil DNA glycosylase (UDG; Rashtchian et al. 1991). This entailed the replacement of oligodeoxynucleotide primers SD2 and SA4 in the second PCR amplification, with SDDU and SADU. The sequences of these primers are as follows:

\section{SDDU: 5'-AUAAGCUUGAUCUCACAAGCTG CACGCTCTAG-3', \\ SADU: 5'-UUCGAGUAGUACUTTCTATTCCT TCGGGCCTGT-3'.}

Complementary primers were also designed for the cloning vector pBluescript IIKS + (Stratagene), surrounding the EcoRV site; these are as follows:

\section{BSDU: $\quad 5^{\prime}$-GAUCAAGCUUAUCGATACCGT CGACC-3', \\ BSAU: 5'-AGUACUACUCGAAUTCCTGCA GCC-3'.}

Ten nanograms of EcoRV-digested pBluescript IIKS + was amplified with BSDU and BSAU. Fifty nanograms of the amplified, linearized plasmid was mixed with 50-100 ng of RT-PCR product, and the mixture was digested with 1 unit of UDG (GIBCO-BRL) at $37^{\circ} \mathrm{C}$ in a $10-\mu \mathrm{l}$ volume of $1 \times \mathrm{PCR}$ buffer. The digested and annealed products were immediately transformed into Escherichia coli DH5 $\alpha$ host. UDG cloning streamlined the procedure and completely eliminated a significant frequency of clone chimerism. Clones from each pool were picked, propagated, frozen, and stored in 96-well microtiter plates. Sequencing was performed using the method of Sanger et al. (1977). Sequences were automatically read using a Millipore Bioimage DNA sequence film reader operating on a Sun Sparc Station. Sequence data base comparisons were performed using the BLAST network service of the National Center for Biotechnology Information (Altschul et al. 1990). The sequences have been deposited in Genbank with the following accession numbers: H55062-H55737.

\section{ACKNOWLEDGMENTS}

We thank Drs. M. Duyao, M.K. McCormick, D.J. Munroe, and $\mathrm{M}$. Lovett for helpful comments, discussion, and critical reading of the manuscript. We also thank the Lawrence Livermore National Laboratory for providing the flow-sorted chromosome 22 cosmid library. This work was supported by grants HG00672 (A.J.B.) and HG00169 (J.F.G.) from the National Institutes of Health.

The publication costs of this article were defrayed in part by payment of page charges. This article must therefore be hereby marked "advertisement" in accordance with 18 USC section 1734 solely to indicate this fact.

\section{REFERENCES}

Adams, M.D., J.M. Kelley, J.D. Gocayne, M. Dubnick, M.H. Polymeropoulos, H. Xiao, C.R. Merril, A. Wu, B. Olde, R.F. Moreno, A.R. Kerlavage, W.R. McCombie, and J.C. Venter. 1991. Complementary DNA sequencing: Expressed sequence tags and human genome project. Science 252: 1651-1656.

Adams, M.D., M. Dubnick, A.R. Kerlavage, R. Moreno, J.M Kelley, T.R. Utterback, J.W. Nagle, C. Fields, and J.C. Venter. 1992. Sequence identification of 2,375 human brain genes. Nature 355: 632-634.

Altschul, S.F., W. Gish, W. Miller, E. Myers, and D.J. Lippman. 1990. Basic local alignment search tool. J. Mol. Biol. 215: 403-410.

Auch, D. and M. Reth. 1990. Exon trap cloning: Using PCR to rapidly detect and clone exons from genomic DNA fragments. Nucleic Acids Res. 18: 6743-6744.

Bischoff, F.R., H. Krebber, T. Kempf, I. Hermes, and H. Ponstingl. 1995. Human RanGTPase-activating protein RanGAP1 is a homologue of yeast Rna1p involved in mRNA processing and transport. Proc. Natl. Acad. Sci. 92: $1749-1753$.

Buckler, A.J., D.D. Chang, S.L. Graw, J.D. Brook, D.A. Haber, P.A. Sharp, and D.E. Housman. 1991. Exon amplification: A strategy to isolate mammalian genes based on RNA splicing. Proc. Natl. Acad. Sci. 88: 4005-4009.

Cachon-Gonzalez, M.B., S. Fenner, J.M. Coffin, C. Moran, S. Best., and J.P. Stoye. 1994. Structure and expression of the hairless gene of mice. Proc. Natl. Acad. Sci. 91: 7717-7721.

Church, D.M., A.C. Rogers, S.L. Graw, D.E. Housman, J.F. Gusella, and A.J. Buckler. 1993. Identification of human chromosome 9 specific genes using exon amplification. Human Mol. Genet. 2: 1915-1920.

Church, D.M., C.J. Stotler, J.L. Rutter, J.R. Murrell, J.A. Trofatter, and A.J. Buckler. 1994. Isolation of genes from complex sources of mammalian genomic DNA using exon amplification. Nature Genet. 6: 98-105. 


\section{CHROMOSOME 22 EXON LIBRARY}

Cohen, D., I. Chumakov, and J. Weissenbach. 1993. A first-generation physical map of the human genome. Nature 366: 698-701.

Corbo, L., J.A. Maley, D.L. Nelson, and C.T. Caskey. 1990. Direct cloning of human transcripts with HnRNA from hybrid cell lines. Science 249: 652-655.

Delmas, V., D.G. Stokes, and R.P. Perry. 1993. A mammalian DNA-binding protein that contains a chromodomain and an SNF2/SWI2-like helicase domain. Proc. Natl. Acad. Sci. 90: 2414-2418.

Del Mastro, R., L. Wang, A.D. Simmons, T.D. Gallardo, G.A. Clines, J.A. Ashley, C.J. Hilliard, J.J. Wasmuth, J.D. McPherson, and M. Lovett. 1995. Human chromosome-specific cDNA libraries: New tools for gene identification and genome annotation. Genome Res. 5: $185-194$

Duyk, G.M., S. Kim, R.M. Myers, and D.R. Cox. 1990. Exon trapping: A genetic screen to identify candidate transcribed sequences in cloned mammalian genomic DNA. Proc. Natl. Acad. Sci. 87: 8995-8999.

Green, E. D. and M.V. Olson. 1990. Systematic screening of yeast artificial-chromosome libraries by use of the polymerase chain reaction. Proc. Natl. Acad. Sci.

87: 1213-1217.

Hästbacka, J., A. de la Chapelle, M. Mahtani, G. Clines, M.P. Reeve, M. Daly, B. Hamilton, K. Kusumi, B. Trivedi, A. Weaver, M. Lovett, A. Buckler, I. Kaitila, and E.S. Lander. 1994. The diastrophic dysplasia gene encodes a novel sulfate transporter: Positional cloning by fine-structure linkage disequilibrium mapping. Cell 78: 1073-1087.

Hastie, N.D. and J.O. Bishop. 1976. The expression of three abundance classes of messenger RNA in mouse tissues. Cell 9: 761-774.

The Huntington's Disease Collaborative Research Group. 1993. A novel gene containing a trinucleotide repeat that is expanded and unstable on Huntington's disease chromosomes. Cell 72: 971-983.

James, T.C. and S.C.R. Elgin. 1986. Identification of a nonhistone chromosomal protein associated with heterochromatin in Drosophila melanogaster and its gene. Mol. Cell. Biol. 6: 3862-3872.

Jones, K.W., M. Chevrette, M.H. Shapero, and R.E.K. Fournier. 1992. Generation of region and species-specific expressed gene probes from somatic cell hybrids. Nature Genet. 1: 278-283.

Khan, A.S., A.S. Wilcox, M.H. Polymeropoulos, J.A. Hopkins, T.J. Stevens, M. Robinson, A.K. Orpana, and J.M. Sikela. 1992. Single pass sequencing and physical and genetic mapping of human brain cDNAs. Nature Genet. 2: 180-185.

Lehmeier, T., V.A. Raker, H. Hermann, and R. Luehrmann. 1994. cDNA cloning of the Sm proteins D2 and D3 from human small nuclear ribonucleoproteins: Evidence for a direct D1-D2 interaction. Proc. Natl. Acad. Sci. 91: 12317-12321.

Lichter, P., S.A. Ledbetter, D.H. Ledbetter, and D.C. Ward. 1990. Fluorescence in situ hybridization with Alu and $\mathrm{L} 1$ polymerase chain reaction probes for rapid characterization of human chromosomes in hybrid cell lines. Proc. Natl. Acad. Sci. 87: 6634-6638.

Liu, P., R. Legerski, and M.J. Siciliano. 1989. Isolation of human transcribed sequences from human-rodent somatic cell hybrids. Science 246: 813-815.

Lovett, M., J. Kere, and L.M. Hinton. 1991. Direct selection: A method for the isolation of cDNAs encoded by large genomic fragments. Proc. Natl. Acad Sci 88: $9628-9632$.

Messmer, S., A. Franke, and R. Paro. 1992. Analysis of the functional role of the Polycomb chromo domain in Drosophila melanogaster.Genes \& Dev. 6: 1241-1254.

Munroe, D.J., R, Loebbert, E. Bric, T. Whitton, D. Prawitt, D. Vu, A. Buckler, A. Winterpracht, B. Zabel, and D.E. Housman. 1995. Systematic screening of an arrayed cDNA library by PCR. Proc. Natl. Acad. Sci.

92: 2209-2213.

Okubo, K., N. Hori, R. Matoba, T. Niiyama, A. Fukushima, Y. Kojima, and K. Matsubara. 1992. Large scale cDNA sequencing for analysis of quantitative and qualitative aspects of gene expression. Nature Genet. 2: 173-179.

Paro, R. and D.S. Hogness. 1991. The Polycomb protein shares a homologous domain with a heterochromatin-associated protein of Drosophila. Proc. Natl. Acad. Sci. 88: 263-267.

Parimoo, S., S.R. Patanjali, H. Shulka, D.D. Chaplin, and S.M. Weissman. 1991. cDNA selection: Efficient PCR approach for the selection of cDNAs in large genomic DNA fragments. Proc. Natl. Acad. Sci. 88: 9623-9627.

Pemble, S., K.R. Schroeder, S.R. Spencer, D.J. Meyer, E. Hallier, H.M. Bolt, B. Ketterer, and J.B. Taylor. 1994. Human glutathione S-transferase theta (GSTT1): cDNA cloning and the characterization of a genetic polymorphism. Biochem. J. 300: 271-276.

Rashtchian, A., G.W. Buchman, D.M. Schuster, and M. Berninger. 1991. Uracil DNA glycosylase-mediated cloning of polymerase chain reaction-amplified DNA: Application to genomic and cDNA cloning. Anal. Biochem. 206: 91-97.

Sanger, F., S. Nicklen, and A.R. Coulson. 1977. DNA sequencing with chain terminating inhibitors. Proc. Natl. Acad. Sci. 74: 5463-5467.

Singh, P.B., J.R. Miller, J. Pearce, R. Kothary, R.D. Burton, R. Paro, T.C. James, and S.J. Gaunt. 1991. A. sequence motif found in a Drosophila heterochromatin protein is 


\section{TROFATTER ET AL.}

conserved in animals and plants. Nucleic Acids Res. 19: 789-794.

Tanihara, H., K. Sano, R.L. Heimark, T. St.John, and S. Suzuki. 1994. Cloning of five cadherins clarifies characteristic features of cadherin extracellular domain and provides further evidence for two structurally different types of cadherin. Cell Adhesion Commun. 2: $15-26$.

Trofatter, J.A., M.M. MacCollin, J.L. Rutter, J.R. Murrell, M.P. Duyao, D.M. Parry, R. Eldridge, N. Kley, A.G.

Menon, K. Pulaski, V. Haase, C. Ambrose, D. Munroe, C. Bove, J.L. Haines, R.L. Martuza, M.E. MacDonald, B.R. Seizinger, M.P. Short, A.J. Buckler, and J.F. Gusella. 1993. A novel moesin-, ezrin-, radixin-like gene is a candidate for the neurofibromatosis 2 tumor suppressor. Cell 72: 791-800.

Vidal, S.M., D. Malo, K. Vogan, E. Skamene, and P. Gros. 1993. Natural resistance to infection with intracellular parasites: Isolation of a candidate for Bcg. Cell 73: $469-485$.

Vulpe, C., B. Levinson, S. Whitney, S. Packman, and J. Gitschier. 1993. Isolation of a candidate gene for Menkes disease and evidence that it encodes a copper-transporting ATPase. Nature Genet. 3: 7-13.

Walker, A.P., F. Muscatelli, and A.P. Monaco. 1993. Isolation of the human Xp21 glycerol kinase gene by positional cloning. Hum. Mol. Genet. 2: 107-114.

Wong, K. and L. Cantley. 1994. Cloning and characterization of a human phosphatidylinositol 4-kinase. J. Biol. Chem. 269: 28878-28884.

Received June 15, 1995; accepted in revised form September $22,1995$. 


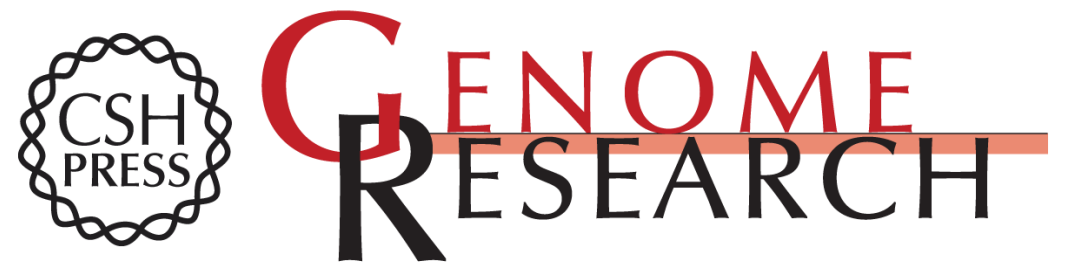

\section{An expression-independent catalog of genes from human chromosome 22.}

J A Trofatter, K R Long, J R Murrell, et al.

Genome Res. 1995 5: 214-224

Access the most recent version at doi:10.1101/gr.5.3.214

References This article cites 40 articles, 21 of which can be accessed free at:

http://genome.cshlp.org/content/5/3/214.full.html\#ref-list-1

\section{License}

Email Alerting Receive free email alerts when new articles cite this article - sign up in the box at the Service top right corner of the article or click here.

\section{Affordable, Accurate Sequencing.}

To subscribe to Genome Research go to:

https://genome.cshlp.org/subscriptions 